\title{
Erratum
}

\section{Superconformal Current Algebras and their Unitary Representations}

Victor G. $\mathrm{Kac}^{1}$ and Ivan T. Todorov ${ }^{2}$

1 Department of Mathematics, M.I.T., Cambridge, MA 02139, USA

2 Institute of Nuclear Research and Nuclear Energy,

Bulgarian Academy of Sciences, BG-1113 Sofia, Bulgaria

Commun. Math. Phys. 102, 337 (1985)

A trivial error slipped into Sect. 6 of the paper. It should be corrected as follows. The range of $m$ in Eq. (6.1) is actually $m=0,1,2, \ldots$ Equations (6.2), (6.4), and (6.5) should read:

$$
\begin{gathered}
\left(\mu_{0}=\mathrm{m}-2 \mathrm{I}, \mu_{1}=2 I\right) \oplus(0,0) \quad(2 I=0,1, \ldots, m), \\
\left(\lambda_{\mathrm{SU}(2)}, \lambda_{\mathrm{SU}(2)}\right)=(m+2,2), c_{G}=\frac{3 m}{m+2}+3, \\
\lambda_{H}=m+4, c_{H}=3 \frac{m+2}{m+4}+\frac{3}{2} .
\end{gathered}
$$

The last sentence in Sect. 6 (before Acknowledgements) should be deleted.

Thus, all admissible central charges of the super Virasoro algebra occur. Moreover, it has been shown recently independently by P. Goddard, A. Kent, and D. Olive and by V. Kac and M. Wakimoto, that a more detailed analysis of this construction yields all allowed (by [14]) values $\Delta_{[\mu]}$ as well. (These authors, and independently A. Tsuchiya and Y. Kanie, showed that a similar result holds for the Virasoro algebra.)

Communicated by A. Jaffe

Received November 19, 1985 